\title{
Genetic and genomic analysis of acute lymphoblastic leukemia in older adults reveals a distinct profile of abnormalities: analysis of 210 patients from the UKALL14 and UKALL60+ clinical trials
}

Thomas Creasey, Emilio Barretta, ${ }^{1}$ Sarra L. Ryan, ${ }^{1}$ Ellie Butler, Amy A. Kirkwood, ${ }^{2}$ Daniel Leongamornlert, ${ }^{3}$ Elli Papaemmanuil, ${ }^{4}$ Pip Patrick, ${ }^{2}$ Laura Clifton-Hadley, ${ }^{2}$ Bela Patel, ${ }^{5}$ Tobias Menne, ${ }^{6}$ Andrew K. McMillan, ${ }^{7}$ Christine J. Harrison, ${ }^{1}$ Clare J. Rowntree, ${ }^{8}$ Nick Morley, ${ }^{9}$ David I. Marks, ${ }^{10}$ Adele K. Fielding ${ }^{11}$ and Anthony V. Moorman ${ }^{1}$

\footnotetext{
'Leukaemia Research Cytogenetics Group, Translational and Clinical Research Institute, Newcastle University, Newcastle upon Tyne, UK; ${ }^{2}$ Cancer Research UK \& UCL Cancer Trials Centre, UCL Cancer Institute University College London, London, UK; ${ }^{3}$ Sanger Institute, Cambridge, UK; ${ }^{4}$ Memorial Sloan Kettering Cancer Center, New York, NY, USA; ${ }^{5}$ Department of Haematology, Queen Mary University of London, London, UK; ${ }^{6}$ Northern Centre for Cancer Care, Newcastle-upon-Tyne Hospitals NHS Foundation Trust, Newcastle upon Tyne, UK; ${ }^{7}$ Department of Haematology, Nottingham University Hospitals NHS Trust, Nottingham, UK; ${ }^{8}$ Department of Haematology, Cardiff and Vale University Health Board, Cardiff, UK; ${ }^{9}$ Department of Haematology, Sheffield Teaching Hospitals NHS Foundation Trust, Sheffield, UK; ${ }^{10}$ Department of Haematology, University Hospitals Bristol and Weston NHS Foundation Trust, Bristol, UK and ${ }^{11} \mathrm{UCL}$ Cancer Institute, University College London, London, UK
}

\author{
Correspondence: \\ T. Creasey \\ tom.creasey@ncl.ac.uk \\ A.V. Moorman \\ anthony.moorman@ncl.ac.uk \\ Received: $\quad$ May 17, 2021. \\ Accepted: $\quad$ September 9, 2021. \\ Prepublished: November 18, 2021. \\ https://doi.org/10.3324/haematol.2021.279177 \\ (2022 Ferrata Storti Foundation \\ Published under a CC-BY-NC license @( $) \Theta$
}

\begin{abstract}
Despite being predominantly a childhood disease, the incidence of acute lymphoblastic leukemia (ALL) has a second peak in adults aged 60 years and over. These older adults fare extremely poorly with existing treatment strategies and very few studies have undertaken a comprehensive genetic and genomic characterization to improve prognosis in this age group. We performed cytogenetic, single nucleotide polymorphism (SNP) array and next-generation sequencing (NGS) analyses on samples from 210 patients aged $\geq 60$ years from the UKALL14 and UKALL60+ clinical trials. BCR-ABL1-positive disease was present in 26\% (55/210) of patients, followed by low hypodiploidy/near triploidy in 13\% (28/210). Cytogenetically cryptic rearrangements in CRLF2, ZNF384 and MEF2D were detected in $5 \%, 1 \%$ and $<1 \%$ of patients, respectively. Copy number abnormalities were common and deletions in ALL driver genes were seen in $77 \%$ of cases. IKZF1 deletion was present in 51\% (40/78) of samples tested and the IKZF1plus profile was identified in over a third (28/77) of cases of B-cell precursor ALL. The genetic good-risk abnormalities high hyperdiploidy (n=2), ETV6-RUNX1 (no cases) and ERG deletion (no cases) were exceptionally rare in this cohort. RAS pathway mutations were seen in $17 \%(4 / 23)$ of screened samples. KDM6A abnormalities, including biallelic deletions, were discovered in 5\% (4/78) of SNP arrays and 9\% (2/23) of NGS samples, and represent novel, potentially therapeutically actionable lesions using EZH2 inhibitors. Outcome remained poor with 5-year event-free and overall survival rates of $17 \%$ and $24 \%$, respectively, across the cohort, indicating a need for novel therapeutic strategies.
\end{abstract}

\section{Introduction}

Acute lymphoblastic leukemia (ALL) presents most commonly in early childhood. However, the disease has a bimodal incidence with a second smaller peak in adults aged 60 years old and over. $^{2}$ Optimal care of these older adults ( $\geq 60$ years) remains an area of unmet clinical need. Although they account for only $30-35 \%$ of diagnoses each year in adults, around $60 \%$ of disease-related deaths occur within this age group, and they are the only ALL patients not to have benefitted from the stepwise improvements in prognosis driven through successive clinical trials in children and younger adults. ${ }^{2,3}$

Primary chromosomal abnormalities are one of the hallmarks of ALL and greatly influence treatment decisions and prognosis. ${ }^{4,5}$ Although $B C R-A B L 1$ is well recognized as the most common genetic subgroup in adult ALL, ${ }^{5}$ to date, only limited biological characterization of older patients beyond the conventionally defined risk groups has been performed. One recent study provided a comprehensive genomic profile of 1,988 subjects with $B$-cell precursor (BCP)-ALL using a combination of transcrip- 
tome, whole genome and exome sequencing and identified 23 genetic subtypes. ${ }^{6}$ Despite this impressive cohort, only 103 patients aged 60 years and over at diagnosis were included. Such efforts focused on older individuals are needed to improve prognostication and to identify novel therapeutic targets. ${ }^{7,8}$

A proportion of patients do not harbor a cytogenetically visible disease-defining lesion, but have a gene expression profile similar to that of $B C R-A B L 1$-positive disease (Ph-like/BCR-ABL-like ALL). ${ }^{9,10}$ Approximately $50 \%$ of children and young adults with this entity have cytogenetically-cryptic IGH-CRLF2 or P2RY8-CRLF2 rearrangements, which activate JAK-STAT signaling. ${ }^{11,12}$ Other recurrent gene rearrangements include $A B L$-class fusions (affecting $A B L 1, A B L 2$, PDGFRB or CSF1R) in $9-13 \%$ of $\mathrm{Ph}-$ like cases and the JAK-STAT pathway activating rearrangements of JAK2 or EPOR in $7-10 \%$ and $3-6 \%$ of patients, respectively. ${ }^{11,12}$ To date, most studies have focused on pediatric and young adult ( $<60$ years) cohorts, ${ }^{11,13}$ although one study found the Ph-like signature in $24 \%$ of older BCP-ALL patients in a restricted subgroup lacking large scale aneuploidy. ${ }^{2}$ Separately, ZNF384 and MEF2D rearrangements have been reported in $2-6 \%$ of pediatric BCP-ALL cases, and form distinct clinical entities. $^{14,15}$

Focal copy number abnormalities (CNA) frequently target genes that are involved in B-cell development or cell cycle regulation These secondary abnormalities drive transformation of a pre-leukemic clone into overt disease and include deletions of EBF1 on $5 q 33.3$, IKZF1 on 7p12.2, CDKN2A and CDKN2B on 9p21.3, PAX5 on 9p13.2, ETV6 on 12p13.2, BTG1 on $12 \mathrm{q} 21.33$ and RB1 on $13 \mathrm{q} 14.2 .^{16}$ Importantly, particular combinations of CNA have an im-

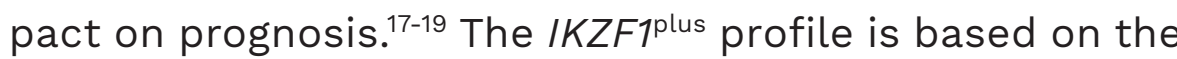
co-occurrence of IKZF1 deletion with deletions of $C D K N 2 A, C D K N 2 B, P A X 5$ or the pseudoautosomal region 1 (PAR1) on Xp22.33/Yp11.31 (resulting in P2RY8-CRLF2 fusion) in the absence of ERG deletion. ${ }^{17}$ This copy number profile is associated with a significantly poorer outcome in childhood ALL patients, highlighting the prognostic importance of large-scale copy number analyses.

To date, profiling the genetic and genomic landscape of ALL has been primarily restricted to younger patients with few analyses focused on older individuals. Here, we applied cytogenetic, copy number and next-generation sequencing (NGS) techniques to investigate whether the primary and secondary genetic abnormalities of ALL in older adults are distinct from those encountered in their younger counterparts. We additionally sought to identify novel druggable targets, a particular priority for such patients because of the high toxicity and low success rates of traditional chemotherapeutic approaches in this group. $^{20}$

\section{Methods}

\section{Patients and samples}

Patients aged 60 years and over were identified for genetic profiling studies from two large UK-wide multicenter clinical trials (UKALL14 and UKALL60+). The UKALL14 study is registered as NCT01085617 (https://www.clinicaltrials.gov/ct2/show/NCT01085617), and the UKALL60+ study is registered as NCT01616238 https://clinicaltrials.gov/ct2/show/NCT01616238) (Online Supplementary Methods).

Baseline cytogenetic analyses, typically consisting of a diagnostic karyotype and fluorescence in situ hybridization (FISH) for BCR-ABL1 fusion and KMT2A translocations, were performed in accredited diagnostic genetic laboratories throughout the UK and then centrally reviewed and entered into the Leukaemia Research Cytogenetics Group database.

The study was approved by the institutional review board of each treatment center and all patients gave written informed consent for data collection and genetic studies as specified by the trials' protocols.

\section{Detection of primary genetic subgroups}

Diagnostic karyotype and FISH results from the regional genetic centers were first examined and patients were coded into one of six subgroups: BCR-ABL1, TCF3-PBX1, $K M T 2 A$ fusions, high hyperdiploidy (51-65 chromosomes), low hypodiploidy/near triploidy (HoTr) (30-39 or 60-78 chromosomes) or T-cell ALL (T-ALL). Reverse transcriptase polymerase chain reaction analysis was performed to identify the presence of Bcr-Abl p190, p210 and p230 oncoproteins in BCR-ABL1-positive cases. Next, all BCP-ALL cases lacking a primary chromosomal abnormality, hereafter termed B-other ALL, were identified. B-other cases with available fixed cell samples were further investigated by the Leukaemia Research Cytogenetics Group to determine the occurrence of cytogenetically cryptic abnormalities using dual color break-apart FISH probes for CRLF2, PDGFRB/CSF1R, ABL2, IGH, ZNF384, MEF2D (Cytocell, Cambridge, UK) and JAK2 (Kreatech Diagnostics, Amsterdam, the Netherlands). Separately, multiplex ligation-dependent probe amplification (MLPA) using the IKZF1-P335 kit (MRC Holland, the Netherlands) was performed on cases with available DNA as previously described ${ }^{21}$ and permitted the detection of the P2RY8-CRLF2 fusion that occurs through PAR1 deletion on Xp22.33/Yp11.31.

\section{Copy number analysis}

Single nucleotide polymorphism (SNP) arrays were performed on DNA extracted from diagnostic bone marrow samples obtained at trial enrolment. SNP arrays were performed at the Newcastle Genomics Centre, Newcastleupon-Tyne Hospitals NHS Foundation Trust using the 
Affymetrix Cytoscan HD (Affymetrix, Santa Clara, CA, USA) or Illumina CytoSNP 850k (Illumina, San Diego, CA, USA) arrays according to the manufacturers' protocols.

Deletions in IKZF1, CDKN2A, CDKN2B, PAX5, RB1, ETV6, EBF1 and $B T G 1$ ('driver genes') were specifically identified from the SNP array results, with loss of any part of the gene considered significant. All arm-level and focal CNA were then examined to detect recurrent abnormalities.

\section{Next-generation sequencing}

Separately, targeted NGS analyses were performed on selected samples using a custom SureSelect XT2 target enrichment kit (Agilent, Santa Clara, CA, USA). Samples were selected based on availability of DNA and the presence of CNA by SNP array. The capture library was designed to target either the coding regions or full sequence of 44 genes that are well known to be implicated in ALL (Online Supplementary Table S1). Libraries were prepared in accordance with the manufacturer's protocol and sequenced on the NextSeq 550 (Illumina, San Diego, CA, USA) using 100 bp paired-end chemistry (Online Supplementary Methods).

\section{Survival analysis}

Survival analysis was restricted to patients enrolled in UKALL14 because all these patients received similar intensive treatment with curative intent. ${ }^{22}$ Patients were grouped according to primary chromosomal abnormalities as described previously. ${ }^{23}$ Briefly, patients with complex karyotypes, HoTr or JAK-STAT activating rearrangements were classed as very high risk; patients with any KMT2A fusions were classed as high risk; patients with $B C R-A B L 1$ and other kinase-activating fusions were classed as having tyrosine kinase-activating (TKA) abnormalities; all other BCP-ALL patients were classed as standard risk; and TALL patients were analyzed separately. All $P$-values were two-sided and, because of multiple testing, values $<0.01$ were considered statistically significant. All analyses were performed using Intercooled Stata (StataCorp, College Station, TX, USA) and R version 3.4.3 (http://www.R-project.org).

\section{Results}

\section{Patients' demographics and baseline cytogenetics}

We identified a total of 210 patients aged $\geq 60$ years from the UKALL14 $(n=95)$ and UKALL60+ $(n=115)$ clinical trials. The median age of the patients was 64 years (range, 60$83)$ and $24 \%(n=50)$ were over 70 years at diagnosis. The male:female ratio was $1: 1$. In total, $90 \%(n=189)$ had confirmed BCP-ALL and 5\% $(n=11)$ had T-cell disease. The remaining $5 \% \quad(n=10)$ did not have a diagnostic immunophenotype centrally recorded. Numbers of patients decreased with advancing age but no significant dif- ference was seen in the genetic subgroups represented in different age groups $(P=0.47)$ (Figure $1 \mathrm{~A}, \mathrm{~B})$. The most prevalent abnormality was $B C R-A B L 1$, present in $28 \%$ $(55 / 200)$ of evaluable patients. Of these, the p210, p190 and p230 isoforms were present in 40\% (22/55), 33\% $(18 / 55)$ and $<1 \%(1 / 55)$ of patients, respectively. Two patients had both p190 and p210 isoforms identified and the $\mathrm{Bcr}-\mathrm{Abl}$ isoform was unknown in the remaining $22 \%$ (12/55) patients. Low hypodiploidy/near triploidy (HoTr) was the second most prevalent primary chromosomal abnormality and was identified in 14\% (28/200) of patients (Online Supplementary Table S2) and KMT2A-V rearrangements were discovered in a further $6 \%$ (12/200) of patients. Of the patients with BCP-ALL, 47\% (88/189) did not have a primary chromosomal abnormality identified by routine cytogenetic and FISH analyses performed in regional cytogenetic centers (B-other ALL).

Among the 11 patients with T-ALL, TLX1 $(n=1)$ and TLX3 $(n=1)$ rearrangements were identified. The other nine patients either had no rearrangements identified $(n=5)$ or were not tested $(n=4)$.

Individual patients' demographic, clinical and genetic data are shown in Online Supplementary Table S3.

\section{Gene rearrangements in patients with B-other acute lymphoblastic leukemia}

Patients with B-other ALL included those with normal $(n=21)$, failed $(n=25)$ or complex karyotypes $(n=5)$. Patients with $\operatorname{dic}(9 ; 12)(n=2), I G H$ translocation $(n=5)$ or other nonsubgroup-defining chromosomal abnormalities $(n=30)$ were also included in the B-other category. Fixed cell samples were available for $74 \%(65 / 88)$ of B-other patients and gene rearrangements were identified in 21\% (19/65) (Table 2). Not all samples could be screened for all abnormalities due to lack of availability of material for multiple FISH experiments.

CRLF2 rearrangements were identified in 17\% (8/48) of successfully screened cases. The CRLF2 rearrangement partners were $I G H(n=5), P 2 R Y 8(n=2)$ and unknown $(n=1)$. Two additional patients had $P 2 R Y 8-C R L F 2$ fusion identified by MLPA, through the presence of PAR1 deletion (Table 1). IGH translocations were present in $26 \%$ (14/53) of B-other samples tested. Of these, five cases accounted for patients with IGH-CRLF2 translocations detailed above, and three cases had separate primary genetic abnormalities identified (one ZNF384 translocation and two P2RY8$C R L F 2$ fusions). In the remaining six samples, the $I G H$ partners were CEBPA $(\mathrm{n}=1), \operatorname{CEBPD}(\mathrm{n}=1), \operatorname{CEBPE}(\mathrm{n}=1), B C L 2$ $(\mathrm{n}=1)$ and unknown $(\mathrm{n}=2)$. ZNF384 and MEF2D rearrangements were each identified in $8 \%(3 / 40)$ and $3 \%(1 / 39)$ of screened B-other cases, respectively.

In total, CRLF2, IGH, and ZNF384 rearrangements were present in $5 \%, 3 \%$ and $1 \%$ of the complete patient cohort, respectively (Figure 1C). No variant $A B L 1$ (0/83), PDGFRB 
(0/56), JAK2 (0/53) or $A B L 2(0 / 52)$ rearrangements were detected.

\section{Copy number alterations}

SNP arrays were performed on diagnostic bone marrow samples from 78 of the 210 patients ( 49 from UKALL14 and 29 from UKALL60+) using the Illumina CytoSNP 850k $(n=51)$ and Affymetrix Cytoscan HD $(n=27)$ arrays. The SNP array cohort was reasonably representative of the whole cohort of patients, although BCR-ABL1-positive patients were slightly over-represented (Online Supplementary Table S4).

Deletions were more frequent than gains in all cytogenetic subgroups apart from high hyperdiploidy. Following the exclusion of probable constitutional copy number variations, as described in the Online Supplementary Methods, a median of seven deletions (range, 0-52) and one gain (range, 0-29) were seen per patient sample.

In the $68 / 78$ patients without a primary ploidy shift (defined as HoTr and high hyperdiploidy), large deletions on $9 p$ were the most prevalent arm-level CNA, seen in $22 \%$
(15/68) of cases (Figure 2, Online Supplementary Table S5). An additional copy of the Philadelphia chromosome was present in $12 \%(8 / 68)$ of patients $(26 \%$ of BCR-ABL1-positive cases) and $1 \mathrm{q}$ gains and monosomy 7 were each present in $10 \%(7 / 68)$ of samples.

Of the CNA in known driver genes, IKZF1 deletions were the most frequent abnormality, present in $51 \%(40 / 78)$ of cases. These were focal intragenic deletions in 19 cases, most commonly involving exons 4-7 $(n=11)$ or exons 2-7 $(n=4)$. Rarer IKZF1 deletions involved exons 4-8 $(n=2)$, exons 2-8 $(n=1)$ and one patient had biallelic IKZF1 loss involving exons 2-7 and 2-8. Focal IKZF1 deletions were almost exclusively seen in patients with $B C R-A B L 1(n=13)$ or $C R L F 2$ rearrangements $(n=5)$ (Online Supplementary Table S6). In the remaining cases, IKZF1 loss resulted from monosomy $7(n=16)$ or $\operatorname{del}(7 p)(n=5)$ (Figure 2, Online Supplementary Table S6).

The pattern of gene deletions varied by $B C R-A B L 1$ status with a higher frequency of IKZF1 deletion in BCR-ABL1positive ALL, as previously described, ${ }^{24}$ and a higher frequency of ETV6 and RB1 deletions in BCR-ABL1-negative

Table 1. Clinical and outcome data for all B-other patients with gene rearrangements detected by fluorescence in situ hybridization or multiplex ligation-dependent probe amplification.

\begin{tabular}{|c|c|c|c|c|}
\hline Patient ID & Trial & Abnormality & WCC (x10\%/L) & Outcome \\
\hline 25130 & UKALL14 & IGH-CRLF2 & 33.6 & Died after 1 month \\
\hline 25371 & UKALL14 & IGH-CRLF2 & 47.7 & Alive $>5$ years \\
\hline 28235 & UKALL60 & IGH-CRLF2 & 5.3 & Relapsed and died after 2 years \\
\hline 30102 & UKALL60 & IGH-CRLF2 & Not known & Relapsed and died after 5 months \\
\hline 30299 & UKALL60 & $I G H-C R L F 2$ & Not known & Died after 4 months \\
\hline 25246 & UKALL14 & P2RY8-CRLF2 & 6.3 & Died within 1 month \\
\hline 28039 & UKALL60 & P2RY8-CRLF2 & Not known & Died after 9 months \\
\hline 25552 & UKALL14 & P2RY8-CRLF2 & 2.9 & Died after 4 months \\
\hline 28011 & UKALL14 & P2RY8-CRLF2 & 3.5 & Died after 16 months \\
\hline 30297 & UKALL60 & CRLF2-r & Not known & Alive $>2$ years \\
\hline 30487 & UKALL14 & IGH-CEBPA & 11.7 & Alive after 1 year \\
\hline 25894 & UKALL60 & $I G H-C E B P D$ & 0.8 & Alive $>5$ years \\
\hline 27181 & UKALL14 & IGH-CEBPE & 1.2 & Died after 3 months \\
\hline 27833 & UKALL60 & $I G H-B C L 2$ & 14.5 & Died after 2 years \\
\hline 25907 & UKALL60 & $I G H-r$ & 2 & Died after 1 year \\
\hline 29808 & UKALL60 & $I G H-r$ & Not known & Relapsed and died after 1 year \\
\hline 25451 & UKALL14 & EP300-ZNF384 & 34.2 & Relapsed and died $>5$ years \\
\hline 25235 & UKALL14 & ZNF384-r & 3.5 & Alive $>5$ years \\
\hline 30085 & UKALL60 & ZNF384-r & Not known & Alive $>2$ years \\
\hline 25267 & UKALL14 & MEF2D-r & 1.4 & Alive $>5$ years \\
\hline
\end{tabular}

Outcome of patients with CRLF2 rearrangement was very poor with only $2 / 10$ alive 2 years after diagnosis. In comparison, $2 / 3$ patients with ZNF384 rearrangements were still alive after 5 years with only one relapse that occurred nearly 7 years after diagnosis. ID: identifier; WCC: white blood cell count. 
A

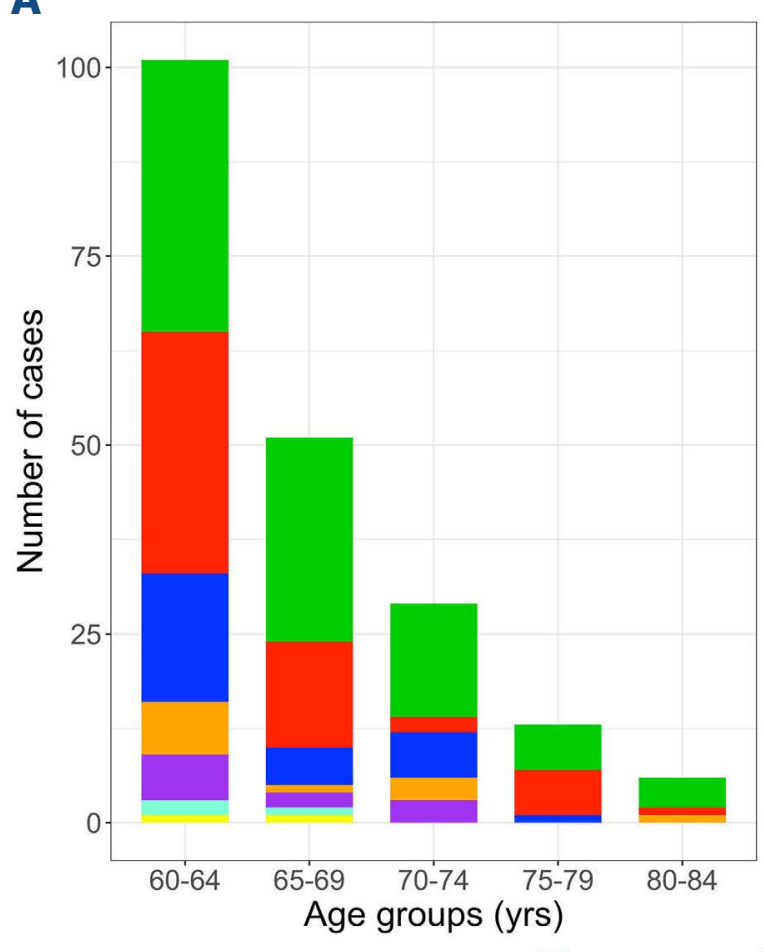

Cytogenetic subgroups B-other
B

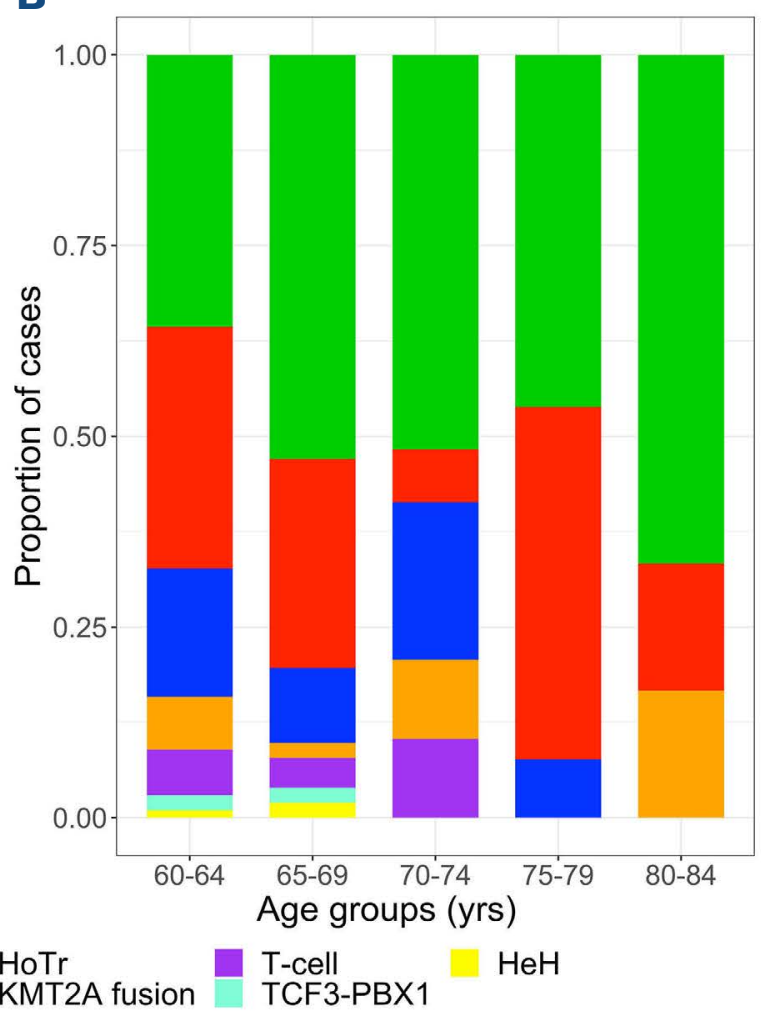

Figure 1. Distribution of primary chromosomal abnormalities by age groups across 210 adults aged $\geq 60$ years. Primary genetic abnormalities shown by 5-year age groups, displayed by number (A) and proportion of cases (B) in each age group. Frequency of final genetic subgroups is shown following screening of B-other acute lymphoblastic leukemia cases for gene rearrangements (C). HoTr: low hypodiploidy/near triploidy; $\mathrm{HeH}$ : high hyperdiploidy; T-cell: T-cell acute lymphoblastic leukemia; Bother: B-cell precursor acute lymphoblastic leukemia in which no primary chromosomal abnormality was identified.

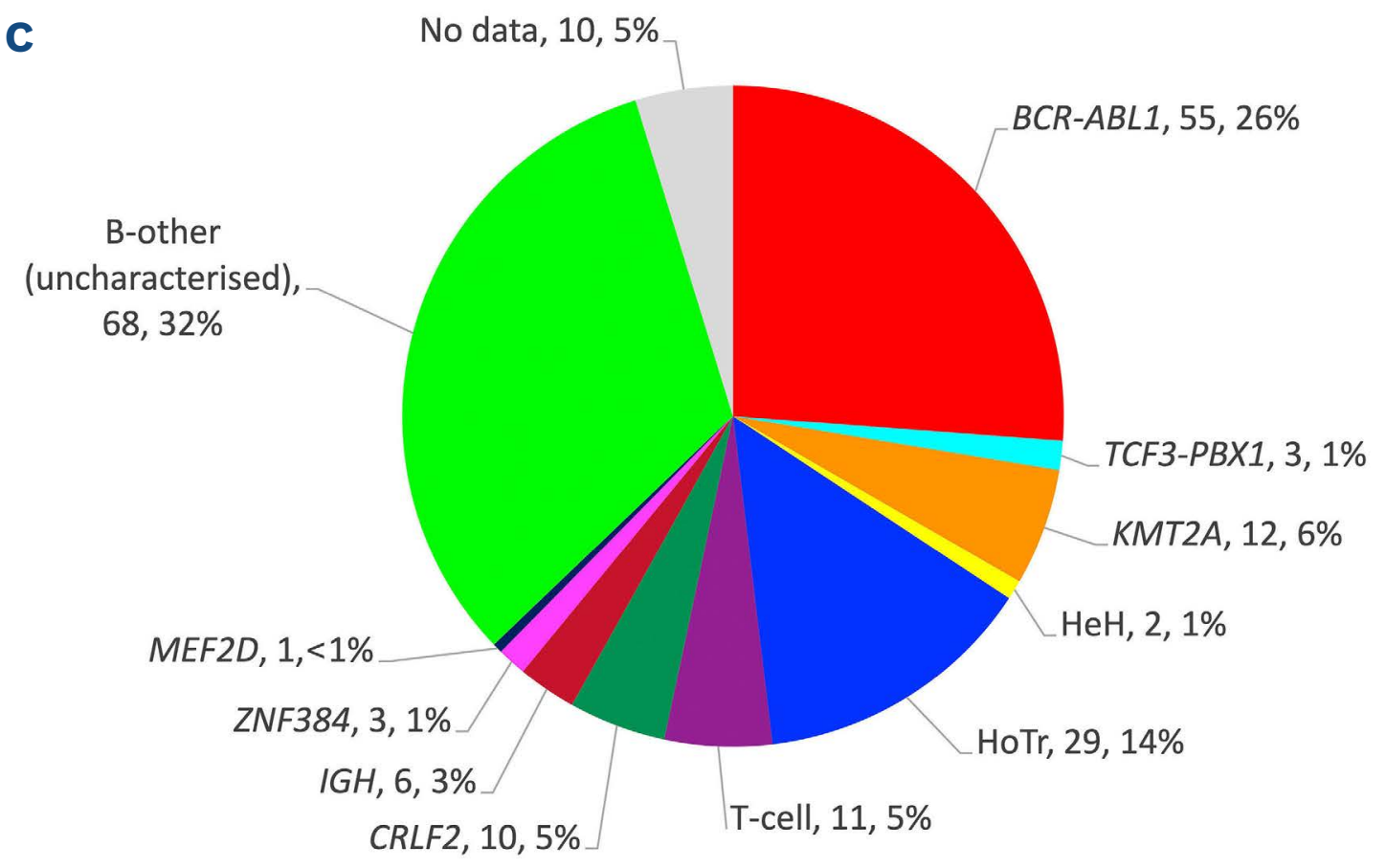

ALL (Table 2). Most deletions were heterozygous with the exception of $C D K N 2 A / B$ which were homozygous in $50 \%$ of cases.

In total, $23 \%(18 / 78)$ of patients had no deletions in driver genes, $18 \%(14 / 78)$ had one deletion, $18 \%(14 / 78)$ had two deletions, $23 \%(18 / 78)$ had three deletions and $18 \%(14 / 78)$ had four or more gene deletions (Online Supplementary Figure S1). IKZF1 deletions in particular co-occurred with other gene deletions much more commonly than in isolation (46\% vs. $5 \%$ ). The $I K Z F 7^{\text {plus }}$ profile ${ }^{17}$ was present in $36 \%$ $(28 / 77)$ of the BCP-ALL samples, specifically in BCR-ABL1positive $(n=13)$, B-other $(n=8)$ and HoTr $(n=7)$ patients.

We detected recurrent focal CNA in several other genes, which to date have not been defined in the pathogenesis of ALL. Focal deletions in LEMD3 on $12 q 14.3$ and KDM6A on Xp11.3 were seen in $6 \%(5 / 78)$ and $5 \%(4 / 78)$ of cases, respectively. Demographic, genetic and outcome features of affected cases are shown in Table 3. LEMD3 deletions ranged from $11-32 \mathrm{~kb}$ in size (Online Supplementary Table S7 and Online Supplementary Figure S2), although they were confined to intron 2 of the gene in three of these. KDM6A deletions ranged from 56-316 kb in size and were homozygous or hemizygous in three of the four cases (Online Supplementary Table S7). Deletion breakpoints for all $\angle E M D 3$ and KDM6A deletions were visually confirmed in IGV in the cases analyzed by NGS.

\section{Mutational landscape}

Twenty-three patients' samples covering all major genetic subgroups (Online Supplementary Table S3) were successfully sequenced using the 44-gene targeted panel. Across these samples, 25 single nucleotide variants and eight in- 


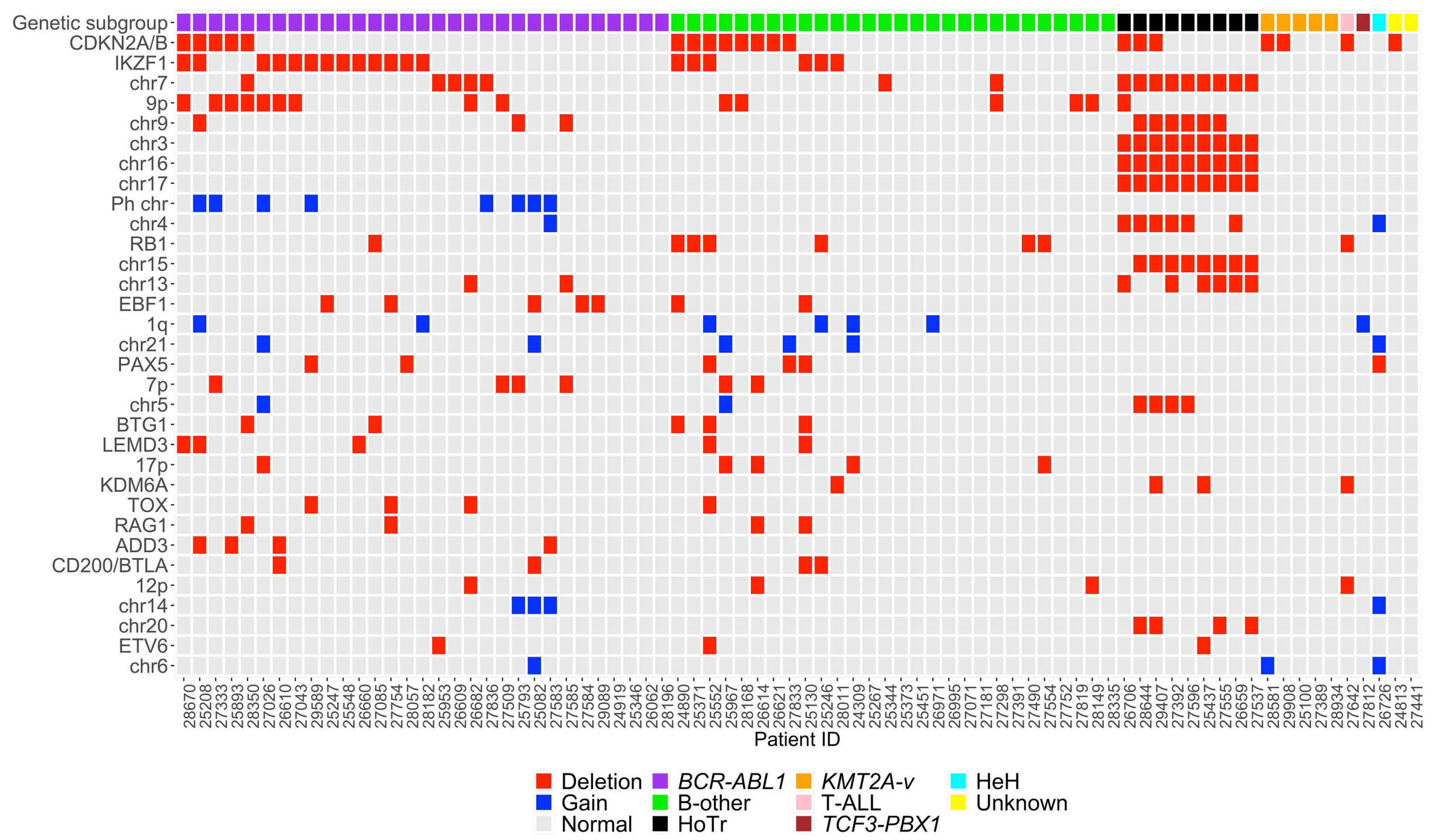

Figure 2. Complete copy number profile based on single nucleotide polymorphism array results from all 78 patients included in the single nucleotide polymorphism array cohort. Only copy number alterations present in at least three patients' samples are displayed. HoTr: low hypodiploidy/near triploidy; HeH: high hyperdiploidy; T-ALL: T-cell acute lymphoblastic leukemia; B-other: B-cell precursor acute lymphoblastic leukemia in which no primary chromosomal abnormality was identified.

Table 2. Frequency of individual deletions in known driver genes split by $B C R-A B L 1$ status. Significant differences identified in rate of IKZF1, ETV6 and RB1 deletions between $B C R-A B L 1$-positive (BCR-ABL $\left.{ }^{+}\right)$and $B C R-A B L 1$ negative $\left(B C R-A B L 1^{-}\right)$cases.

\begin{tabular}{|c|c|c|c|c|c|c|}
\hline \multirow[b]{2}{*}{ Gene } & \multirow[b]{2}{*}{$\begin{array}{l}\text { Cases with } \\
\text { deletion (n) }\end{array}$} & \multicolumn{3}{|c|}{ Deletion frequency by $B C R-A B L 1$ status } & \multirow[b]{2}{*}{$\begin{array}{c}\text { Heterozygous } \\
\text { deletions }\end{array}$} & \multirow[b]{2}{*}{$\begin{array}{c}\text { Homozygous } \\
\text { deletions }\end{array}$} \\
\hline & & $\begin{array}{c}\text { BCR-ABL1 } \\
\text { cases }(n=31)\end{array}$ & $\begin{array}{c}\text { BCR-ABL1- } \\
\text { cases }(n=47)\end{array}$ & P-value & & \\
\hline$I K Z F 1$ & $51 \%(40)$ & $68 \%(21)$ & $40 \%(19)$ & 0.02 & 41 & 2 \\
\hline CDKN2A & $46 \%(36)$ & $39 \%(12)$ & $51 \%(24)$ & 0.36 & 18 & 18 \\
\hline CDKN2B & $46 \%(36)$ & $39 \%(12)$ & $51 \%(24)$ & 0.36 & 21 & 15 \\
\hline PAX5 & $41 \%(32)$ & $48 \%(15)$ & $36 \%(17)$ & 0.35 & 32 & 0 \\
\hline$R B 1$ & $23 \%(18)$ & $10 \%(3)$ & $32 \%(15)$ & 0.03 & 17 & 1 \\
\hline ETV6 & $21 \%(16)$ & $6 \%(2)$ & $30 \%(14)$ & 0.02 & 16 & 0 \\
\hline EBF1 & $21 \%(16)$ & $19 \%(6)$ & $21 \%(10)$ & 1 & 15 & 1 \\
\hline BTG1 & $13 \%(10)$ & $6 \%(2)$ & $17 \%(8)$ & 0.3 & 9 & 1 \\
\hline
\end{tabular}

dels were identified (Figure 3). At least one gene in the NGS panel was mutated in $74 \%$ (17/23) of patients. Pathogenic mutations in the RAS signaling pathway were identified in 17\% (4/23) of cases. KRAS p.G12D and KRAS p.R68W variants were seen in patients with KMT2A and EP300ZNF384 rearrangements, respectively. NRAS p.G12S and p.G12D were present in one B-other case and one patient with unidentified genetic subgroup, respectively. With the exception of the KRAS p.R68W variant, all RAS pathway mutations are reported in the COSMIC database. ${ }^{25}$
Two patients' samples with HoTr were included. Consistent with the underlying chromosomal abnormality, a pathogenic TP53 variant (TP53 p.R282W) was detected in one of these. ${ }^{26,27}$ Two indels in NF1 and an FLT3 missense variant (FLT3 p.V194M) were also seen, the latter being reported as a tolerated passenger mutation in acute myeloid leukemia. ${ }^{28}$

Known pathogenic variants were also seen in JAK2 (JAK2 p.R683T in a patient with IGH-CRLF2), CREBBP (CREBBP p.L1499Q in a patient with IGH-BCL2), and CSF1R (CSF1R 
p.V32G in a patient with $P 2 R Y 8-C R L F 2$ ). Additionally, previously unreported KDM6A mutations were discovered in two $B C R$-ABL1-positive patients (KDM6A p.Y215H and p.K987Q).

We also investigated whether mutations associated with clonal hematopoiesis of indeterminate potential (CHIP) (most commonly affecting DNMT3A, TET2 and ASXL1) were present in older adults with ALL. ${ }^{29,30}$ These are found in $10 \%$ of adults over the age of 65 years without hematologic diseases, but are associated with an increased risk of subsequently developing myelodysplastic syndrome or acute myeloid leukemia. ${ }^{30}$ Overall, these were discovered

Table 3. Demographic, clinical and outcome data of all cases with focal LEMD3 or KDM6A deletions.

\begin{tabular}{|c|c|c|c|c|c|c|}
\hline Gene & Patient ID & $\operatorname{Sex}(M / F)$ & Age (years) & Genetic subgroup & WCC $\left(\times 10^{9} / L\right)$ & Outcome \\
\hline LEMD3 & 25208 & $M$ & 62 & $B C R-A B L 1$ & 205.4 & Alive after 9 years \\
\hline LEMD3 & 25130 & $\mathrm{~F}$ & 62 & IGH-CRLF2 & 33.6 & Died after 1 month \\
\hline LEMD3 & 28670 & $\mathrm{~F}$ & 61 & $B C R-A B L 1$ & 1.6 & Died after 2 months \\
\hline LEMD3 & 26660 & $\mathrm{~F}$ & 62 & $B C R-A B L 1$ & 18.2 & Alive after 7 years \\
\hline LEMD3 & 25552 & $M$ & 61 & P2RY8-CRLF2 & 2.9 & Died after 4 months \\
\hline$K D M 6 A$ & 28011 & M & 61 & B-other & 3.5 & Died after 16 months \\
\hline$K D M 6 A$ & 29407 & $\mathrm{~F}$ & 60 & HoTr & 2.9 & Died after 5 months \\
\hline KDM6A & 25437 & $\mathrm{~F}$ & 64 & $\mathrm{HoTr}$ & 1.4 & Died after 14 months \\
\hline KDM6A & 27642 & $\mathrm{~F}$ & 72 & $T-A L L$ & Not known & Died after 18 months \\
\hline
\end{tabular}

All patients with KDM6A deletions died within 18 months of diagnosis. ID: identifier; M: male; F: female; WCC: white blood cell count; HoTr: low hypodiploidy/near triploidy; T-ALL: T-cell acute lymphoblastic leukemia

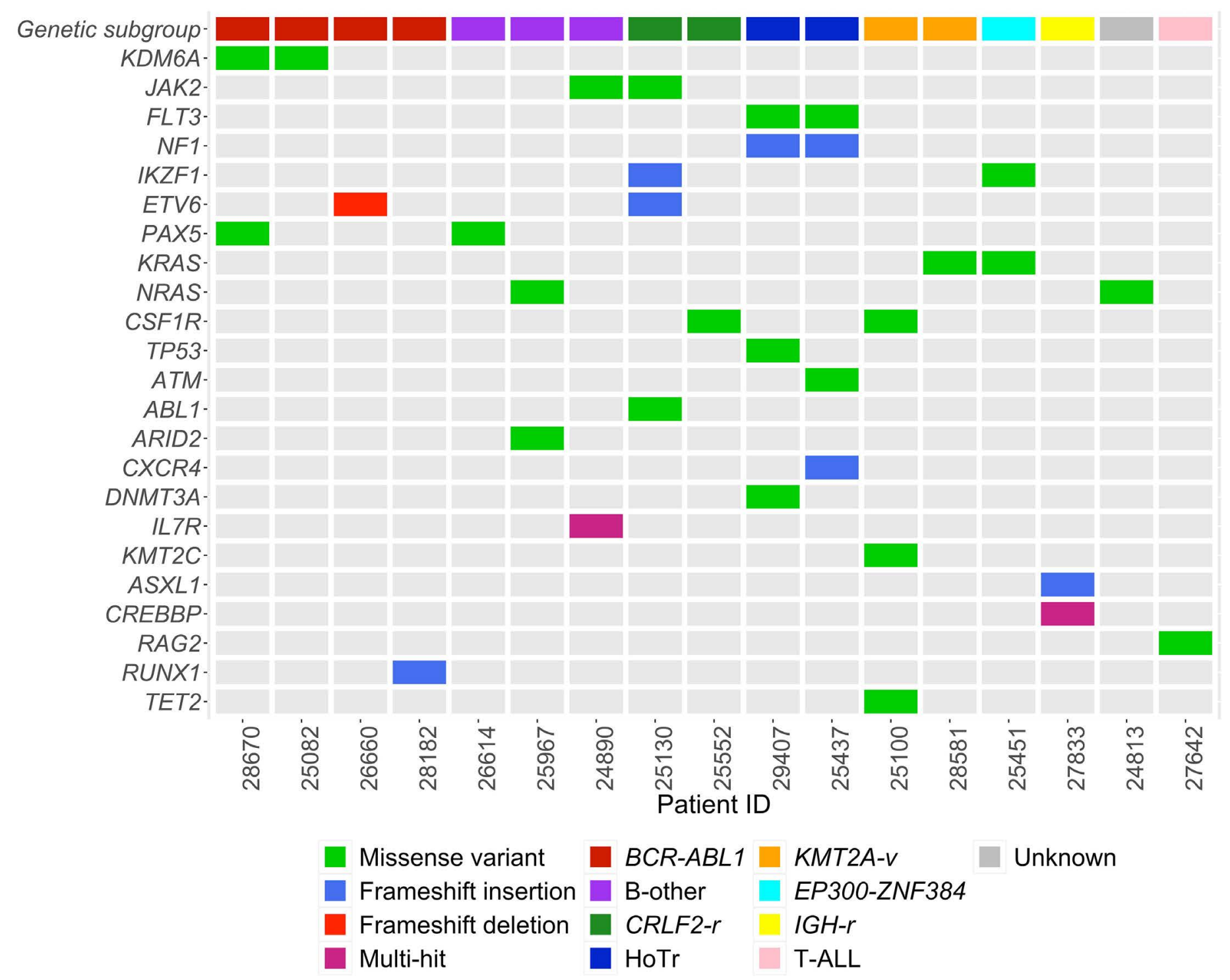

Figure 3. Mutations detected by the 44-gene next-generation sequencing panel in 23 patients. Only patients' samples with at least one mutation are displayed $(n=17)$. In total, 24 single nucleotide variants, seven frameshift insertions and one frameshift deletion were identified. Two genes had both single nucleotide variants and indels within the same case ("multi-hit"). HoTr: low hypodiploidy/near triploidy; T-ALL: T-cell acute lymphoblastic leukemia; B-other: B-cell precursor acute lymphoblastic leukemia in which no primary chromosomal abnormality was identified. 
A

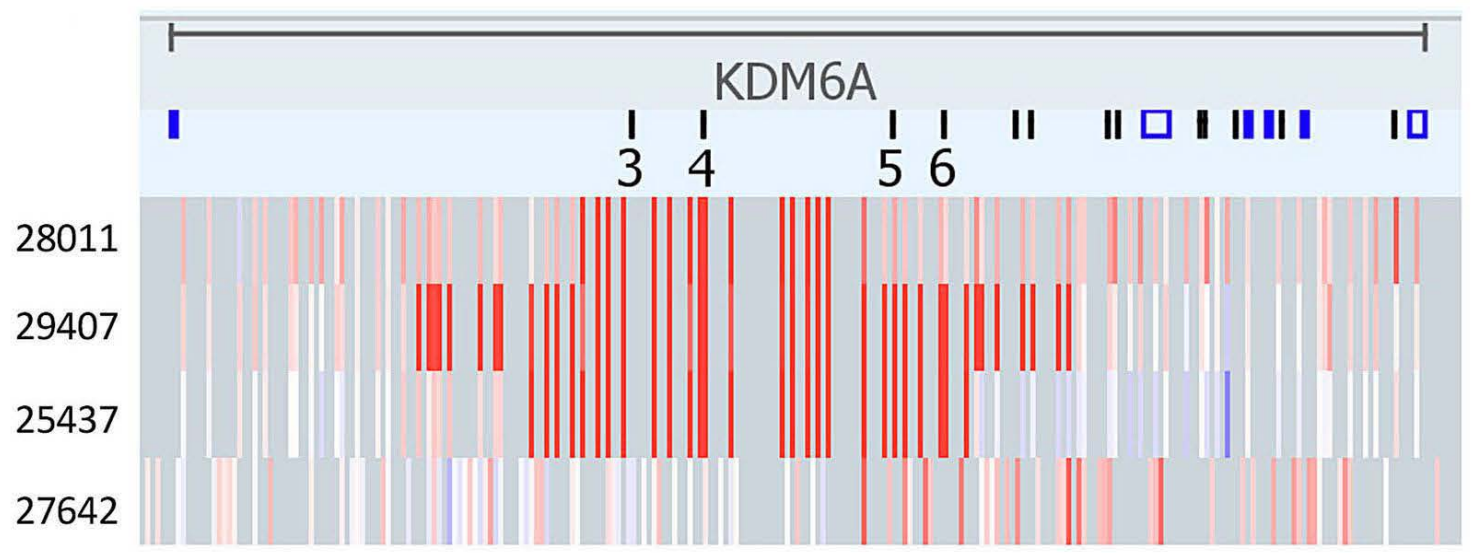

$\mathbf{B}$
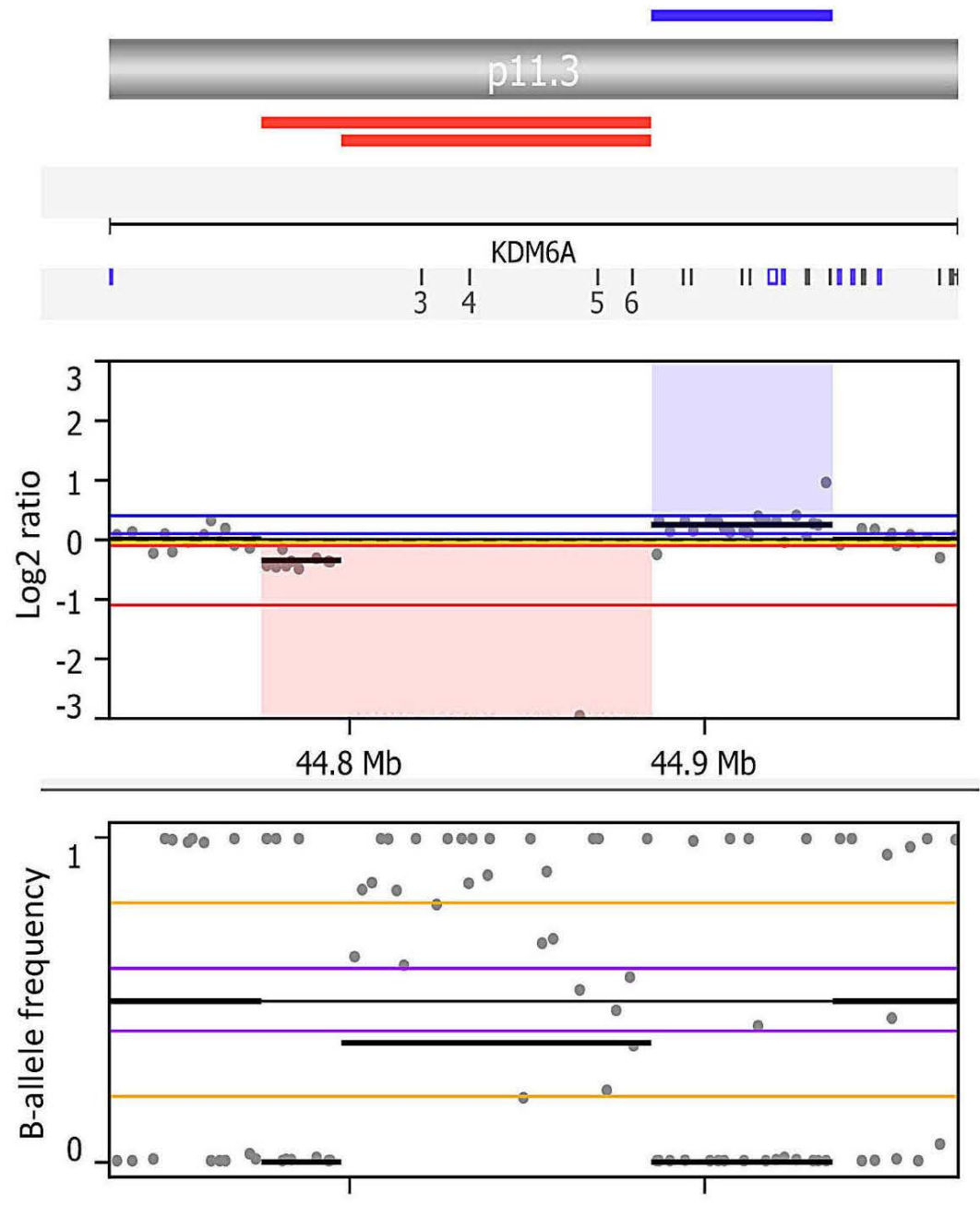

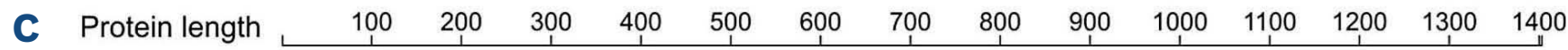

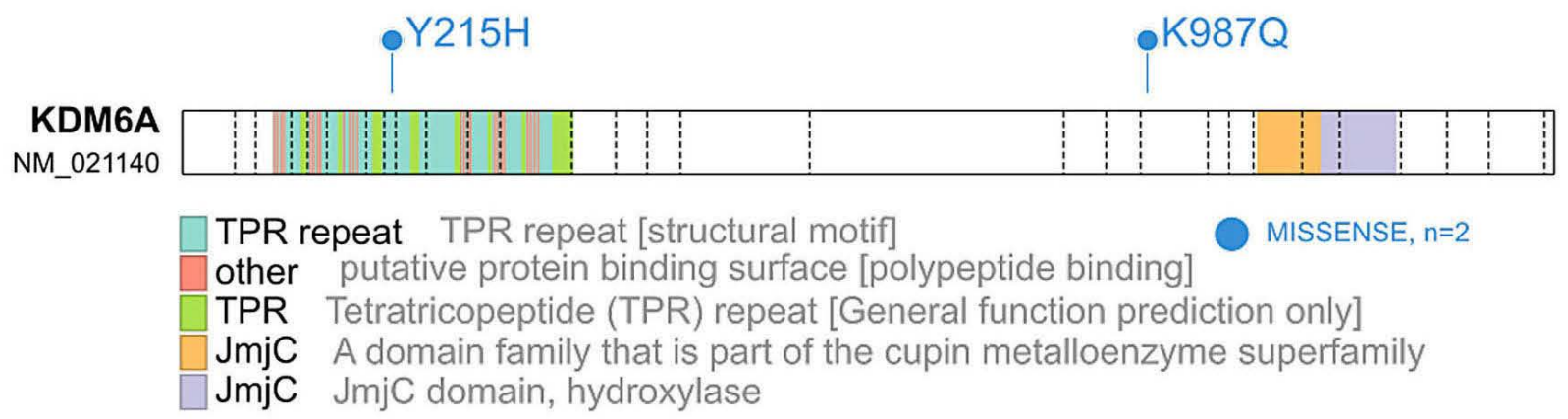

Figure 4. KDM6A aberrations detected by single nucleotide polymorphism array and next-generation sequencing. $K D M 6 A$ deletions identified by single nucleotide polymorphism (SNP) array in four patients' samples (A). Each bar represents a probe on the SNP array. Red colors indicate negative $\log _{2}$ ratio (copy number loss), blue colors represent positive log ratio (copy number gain), and white represents no copy number change. Homozygous KDM6A deletion in patient 25437, demonstrating two slightly distinct KDM6A deletions measuring $110 \mathrm{~kb}$ and $87 \mathrm{~kb}$, and resulting in biallelic loss of exons 3-6 (B). Small gain also noted following segment of homozygous deletion. KDM6A protein plot displaying two mutations detected by next-generation sequencing (C). 
in only $13 \%(3 / 23)$ of cases with single variants in each of DNMT3A, TET2 and ASXL1.

\section{KDM6A alterations}

Overall KDM6A was disrupted in six cases, with focal deletions in 5\% (4/78) of SNP array samples (Table 3) and mutations in $9 \%(2 / 23)$ of NGS samples (Figure 3$)$. Interestingly, the deletions resulted in homozygous or hemizygous KDM6A loss in three of the four cases (Figure 4A, Online Supplementary Table S7). Biallelic KDM6A deletions were seen in the two female patients with HoTr ALL, albeit by two different mechanisms. By cytogenetics and SNP array, patient \#29407 had lost one copy of chromosome $\mathrm{X}$ and had a focal KDM6A deletion in the remaining homologue. In comparison, patient \#25437 had two focal but subtly distinct intragenic KDM6A microdeletions on each $\mathrm{X}$ chromosome (Figure 4B). As KDM6A is not in a pseudoautosomal region, the male patient (\#28011) had a deletion affecting the only KDM6A allele, resulting in hemizygous loss. The KDM6A mutations detected by NGS were present in exons 8 (KDM6A p.Y215H) and 20 (KDM6A p.K987Q) (Figure 5C) and are not reported in the literature although the $\mathrm{SIFT}^{31}$ and Polyphen ${ }^{32}$ in silico prediction tools describe deleterious and probably damaging consequences, respectively, consistent with loss of function. Patients with KDM6A deletions had a poor outcome and all four affected patients died 5-18 months after diagnosis (Table 2). Similarly, the two patients with KDM6A mutations both died within 2 months of diagnosis.

\section{Patients' outcome by genetic subtype}

Outcome data were available for analysis for all 95 UKALL14 patients. Five-year event-free survival and overall survival rates were $17 \%$ and $24 \%$, respectively (Figure 5,
A

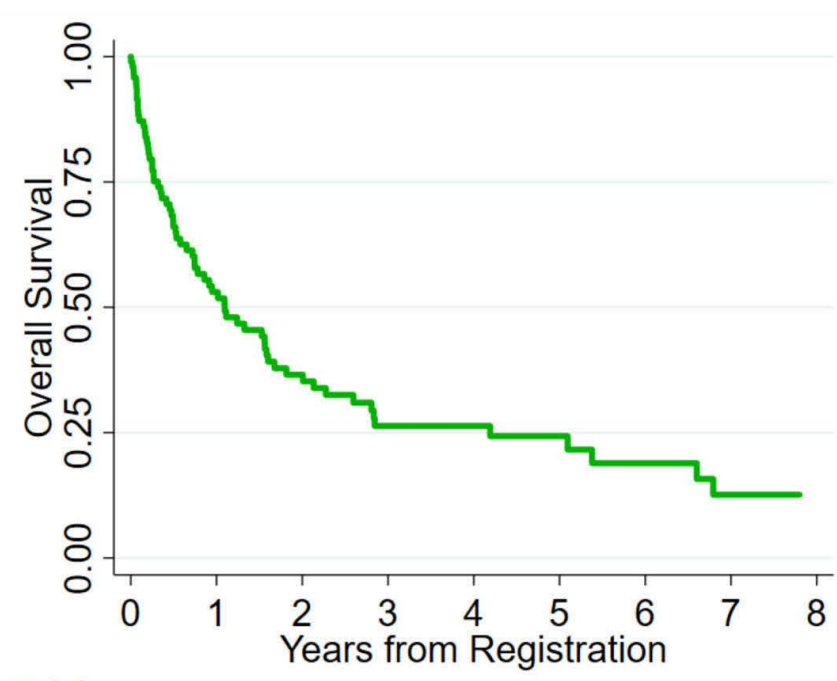

Number at risk $\begin{array}{lllllllll}95 & 43 & 28 & 17 & 14 & 9 & 7 & 4 & 2\end{array}$

C

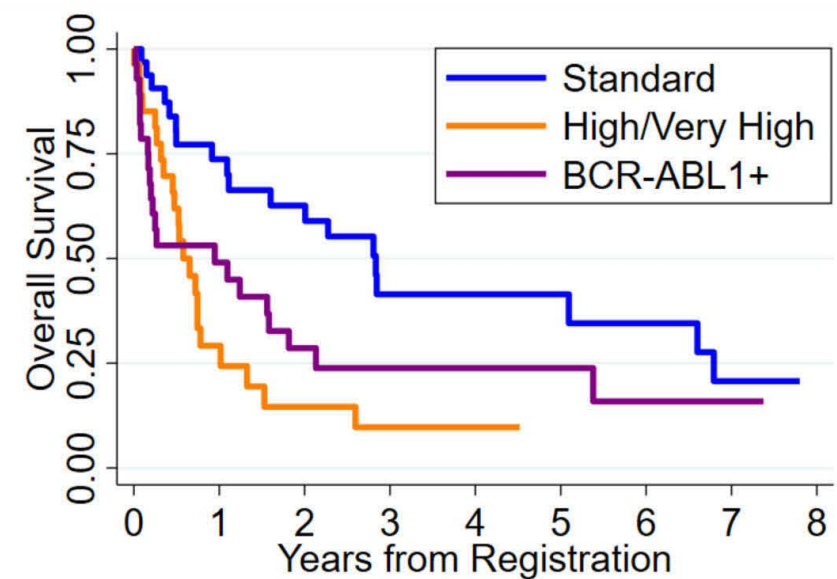

Number at risk

$\begin{array}{lllllllll}\text { Standard } 33 & 21 & 17 & 9 & 7 & 6 & 5 & 3 & 2\end{array}$

High/Very High 28

BCR-ABL1+ 28

$\begin{array}{ccc}6 & 3 & 2 \\ 12 & 7 & 5\end{array}$

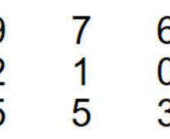

$\begin{array}{llll}6 & 5 & 3 & 2 \\ 0 & 0 & 0 & 0 \\ 3 & 2 & 1 & 0\end{array}$

Figure 5. Overall survival and event-free survival for 95 adults aged $\geq 60$ years recruited into the UKALL14 study. Overall survival (A) and event-free survival (B) for all patients combined; overall survival (C) and event-free survival (D) split by genetic risk group with very high risk and high risk combined into a single group. Patients with complex karyotypes, low hypodiploidy/near triploidy or CRLF2 rearrangements were classed as very high risk; patients with any KMT2A fusions were classed as high risk; patients with $B C R-A B L 1$ and other kinase-activating fusions were classed as tyrosine kinase-activating abnormalities (all $B C R-A B L 1-$ positive in this study); all other patients with B-cell precursor acute lymphoblastic leukemia were classed as standard risk (SR). Patients with SR or BCR-ABL1 had improved overall survival ( $P=0.001)$ and event-free survival $(P=0.002)$ compared to patients with high or very high risk disease. 
Online Supplementary Table S8). Even though the outcome of these older patients was poor there was evidence that tumor genetics remained a strong risk factor (Figure 5), as we have previously demonstrated for younger adults. ${ }^{23} \mathrm{~Pa}-$ tients with standard-risk genetics had the best outcome with $84 \%$ entering remission and 5-year event-free and overall survival rates of $28 \%$ and $41 \%$, respectively. In comparison, over a third of patients with very high-risk genetics did not go into remission and all 28 patients with genetic high-risk or very high-risk disease died within 5 years of diagnosis.

Regarding patients with B-other ALL with gene rearrangements identified, all three patients with ZNF384-rearranged ALL survived more than 2 years from diagnosis whereas six out of eight of those with CRLF2 rearrangements and four out of six with $/ G H$ translocations (excluding CRLF2 partners) died within 2 years (Table 1).

Of the 40 patients with IKZF1 deletions, no significant difference in outcome was identified between those with focal deletions of exons 4-7 (generating the dominant negative IK6 isoform) ${ }^{33}$ compared with patients with other IKZF1 deletions (Online Supplementary Table S9).

\section{Discussion}

To our knowledge, our study provides the largest genetic and genomic characterization to date of older adults with ALL.

The landscape of primary chromosomal abnormalities and CNA is distinct from that observed in children and younger adults. T-ALL was only seen in $5 \%$ of cases, which is less than half the rate seen in younger adults. ${ }^{34}$

Overall, a quarter of patients had $B C R-A B L 1$-positive ALL, although the frequency of this did not increase further over the age of 60 years. This corroborates the findings from a large analysis of three German Multicenter study group for adult ALL (GMALL) trials in which the proportion of $B C R-A B L 1$-positive cases reached a plateau after 45 years of age. ${ }^{35}$ In comparison, our study highlights that HoTr becomes more common with advancing age, such that it is encountered in $<2 \%$ of childhood patients, ${ }^{36} 4-$ $9 \%$ of adults aged $25-60,{ }^{23}$ rising to around $15 \%$ of adults aged 60 years and over in our cohort. Other high-risk cytogenetic subgroups, specifically KMT2A fusions and complex karyotypes were present in $6 \%$ and $3 \%$ of patients, respectively, which are similar rates to those seen in younger adults. ${ }^{5,23}$ We found a lower frequency of CRLF2 rearrangements than found in two USA studies that included older adults.12,37 This could be related to the higher prevalence of Hispanic ancestry in the USA, and the associated inheritance of GATA3 risk alleles, which confer an elevated risk of CRLF2-rearranged ALL. ${ }^{38}$ In comparison to younger patients, ABL-class fusions were notably ab- sent in our cohort. Other studies have similarly identified very low frequencies of $A B L$-class fusions in older individuals. Indeed, only two cases were identified in 103 adults aged 60 years or over in a recent large USA study. ${ }^{6}$ Data from the GMALL group similarly demonstrated a rapid decrease in the frequency of $P h$-like $A L L$ in older age groups. ${ }^{39}$

Although we limited our survival analysis to UKALL14 patients, we highlight that prognosis remains very poor in older adults with ALL (Figure 5). However, consistent with recent studies, patients with ZNF384 rearrangements seemed to have a favorable outcome. ${ }^{23}$

CNA in key genes recurrently disrupted in ALL were discovered in the majority of patients. IKZF1 loss was present in over half of all cases tested by SNP array, occurring in $68 \%$ of $B C R-A B L 1$-positive and $40 \%$ of $B C R-A B L 1$-negative ALL. The high rate of IKZF1 loss in BCR-ABL1-positive ALL is consistent with much of the published literature. ${ }^{24,40}$ However, the frequency of IKZF1 deletion in the older patients with $B C R-A B L 1$-negative ALL was double that reported in younger adults (40\% vs. 19\%). ${ }^{41}$ This discrepancy is at least in part driven by the increased frequency of low hypodiploid cases, as these usually only retain one copy of chromosome 7. However, IKZF1 deletions were still encountered in $36 \%(10 / 28)$ of $B$-other cases. Deletions in other key driver genes in BCP-ALL (CDKN2A/B, PAX5, RB1, ETV6 and EBF1) were also encountered more frequently than in younger patients. ${ }^{23}$

The high-risk IKZF1plus copy number profile was identified in over a third of patients, although its prognostic impact in older adults still needs to be elucidated. Interestingly, no focal ERG deletions, which are associated with a favorable outcome, were detected. ${ }^{42}$ Overall, these data confirm that all genetic biomarkers typically associated with a good prognosis, namely ETV6-RUNX1 fusion, high hyperdiploidy and $E R G$ deletions are exceedingly rare in older adults with ALL, contributing to the adverse outcomes of this population of patients. By virtue of the techniques used, we recognize that we were not able to identify certain novel subgroups, such as the recently described PAX5-driven subtypes ${ }^{6}$ or DUX4 rearrangements, although the latter are associated with ERG deletions in the majority of cases. ${ }^{43}$

Importantly, our study highlighted therapeutically actionable targets that would merit further investigation in older adults. We identified KDM6A deletions and mutations in $5 \%(4 / 78)$ and $9 \%(2 / 23)$ of screened patients, respectively. KDM6A (also known as UTX) on Xp11.3 is an H3K27me3 demethylase, involved in epigenetic regulation through repression of PRC2/EZH2 activity. Recurrent KDM6A mutations have been identified in T-ALL, and have been shown to have gender-specific tumor suppressor effects. ${ }^{44}$ KDM6A escapes $\mathrm{X}$-inactivation in females and therefore retains biallelic expression. Hence, loss of tumor sup- 
pressor function through $K D M 6 A$ abnormalities disproportionately affects males and has been postulated to explain the skewed gender distribution of T-ALL. To our knowledge, our study is the first to demonstrate KDM6A disruption in a significant proportion of older adults with ALL, most of whom had B-cell disease. Most interestingly, we have highlighted homozygous KDM6A deletions in female patients, and no evidence of skewed gender distribution. Loss of KDM6A function, resulting in EZH2 overactivity, has been shown to play an important pathogenic role in urothelial bladder cancer ${ }^{45}$ and functional analyses have demonstrated susceptibility of KDM6A-null cell lines to the Food and Drug Administration-approved EZH2 inhibitor tazemetostat. ${ }^{45}$ Our findings therefore identify a proportion of patients who may respond to EZH2 inhibition, a treatment as yet untested in ALL.

We also discovered recurrent small focal intragenic deletions in $L E M D 3$ in $6 \%(5 / 78)$ of cases. $L E M D 3$ regulates bone morphogenic protein and transforming growth factor $\beta$ signaling and to date has not been implicated in cancer. ${ }^{46}$ The significance of these deletions remains speculative, particularly as some were confined to introns. Recurrent RAS pathway mutations were identified in almost one fifth of patients (4/23), all of whom had BCR$A B L 1$-negative ALL. These activate RAS signaling and are potentially therapeutically actionable through MEK inhibition (e.g., with selumetinib).

Older adults with ALL fare extremely poorly with current chemotherapeutic approaches. Various studies have demonstrated the disproportionate treatment toxicities experienced by this group of patients, leading to treatment omissions or delays..$^{20}$ Our analysis confirms the additional challenges posed by the high proportion of poor-risk genetic subgroups. Moving forward, the comprehensive identification of druggable targets such as KDM6A abnormalities, JAK-STAT-activating rearrangements or RAS pathway mutations presents an opportunity to expand therapeutic options, likely to most benefit this patient population. As a paradigm, significant progress has been made in the management of $B C R-A B L 1$-positive disease through these approaches, culminating in a promising chemotherapy-free protocol. ${ }^{47}$ Further dedicated clinical trials that include comprehensive genomic profiling of older adults, combined with targeted treatments and/or immunotherapy and a reduction in the traditional chemotherapy backbone will be key to improving the dismal outcome of these patients.

\section{Disclosures}

TC was supported by grants from the NIHR Newcastle Biomedical Research Centre and Bright Red. The UKALL14 and UKALL6O+ trials were coordinated by the Cancer Research UK (CRUK) \& UCL Cancer Trials Centre and funded by CRUK (C27995/A9609 and C27995/A13920, respectively). The UKALL60+ trial was also supported by an educational grant from Jazz Pharmaceuticals UK Ltd. This study was supported by research grants from Cancer Research UK (AVM and AKF) and Blood Cancer UK (AVM and CJH).

\section{Contributions}

TC and AVM designed the study. TC, AVM, EB and EB collected and assembled the data. TC, AVM, EB, EB and SLR performed data analysis and interpretation. $C J H$ and $A V M$ were responsible for administrative support. AKF was chief investigator of the UKALL14 and UKALL6O+ clinical trials. $A A K, D L, E P, P P, L C H, B P, T M, A K M, C J R, N M, D I M$, and $A K F$ participated in recruitment of patients and provided study materials. TC and AVM developed the first drafts of the manuscript. All authors contributed to the review and amendments of the manuscript and approved the final version for submission.

\section{Acknowledgments}

The authors thank all the participating sites, local investigators and research teams for their ongoing participation in the study, together with patients who took part in these trials as well as their families. We acknowledge the input of all the scientists and technicians working in the adult ALL MRD laboratory based at UCL. We thank the member laboratories of the UK Cancer Cytogenetic Group for cytogenetic data and material.

\section{Data-sharing statement}

The datasets generated or analyzed during the current study are available in the Online Supplementary Material or from the corresponding author on reasonable request.

\section{References}

1. Inaba $H$, Greaves M, Mullighan CG. Acute lymphoblastic leukaemia. Lancet. 2013;381(9881):1943-1955.

2. Moorman AV, Chilton L, Wilkinson J, et al. A population-based cytogenetic study of adults with acute lymphoblastic leukemia. Blood. 2010;115(2):206-214.

3. Dinmohamed AG, Szabó A, van der Mark M, et al. Improved survival in adult patients with acute lymphoblastic leukemia in the Netherlands: a population-based study on treatment, trial participation and survival. Leukemia. 2016;30(2):310-317.

4. Moorman AV, Ensor HM, Richards SM, et al. Prognostic effect of chromosomal abnormalities in childhood B-cell precursor acute lymphoblastic leukaemia: results from the UK Medical Research Council ALL97/99 randomised trial. Lancet Oncol. 2010;11(5):429-438.

5. Moorman AV, Harrison CJ, Buck GAN, et al. Karyotype is an independent prognostic factor in adult acute lymphoblastic 
leukemia (ALL): analysis of cytogenetic data from patients treated on the Medical Research Council (MRC) UKALLXII/Eastern Cooperative Oncology Group (ECOG) 2993 trial. Blood. 2007;109(8):3189-3197.

6. Gu Z, Churchman ML, Roberts KG, et al. PAX5-driven subtypes of B-progenitor acute lymphoblastic leukemia. Nat Genet. 2019;51(2):296-307.

7. Schultz KR, Pullen DJ, Sather HN, et al. Risk- and responsebased classification of childhood B-precursor acute lymphoblastic leukemia: a combined analysis of prognostic markers from the Pediatric Oncology Group (POG) and Children's Cancer Group (CCG). Blood. 2007;109(3):926-935.

8. Gökbuget N. How I treat older patients with ALL. Blood. 2013;122(8):1366-1375.

9. Mullighan CG, Su X, Zhang J, et al. Deletion of IKZF1 and prognosis in acute lymphoblastic leukemia. N Engl J Med. 2009;360(5):470-480.

10. Den Boer ML, van Slegtenhorst M, De Menezes RX, et al. A subtype of childhood acute lymphoblastic leukaemia with poor treatment outcome: a genome-wide classification study. Lancet Oncol. 2009;10(2):125-134.

11. Roberts KG, Li Y, Payne-Turner D, et al. Targetable kinaseactivating lesions in Ph-like acute lymphoblastic leukemia. $\mathrm{N}$ Engl J Med. 2014;371(11):1005-1015.

12. Roberts KG, Zhaohui G, Debbie P-T, et al. High frequency and poor outcome of Philadelphia chromosome-like acute lymphoblastic leukemia in adults. J Clin Oncol. 2017;35(4):394-401.

13. Roberts KG, Morin RD, Zhang J, et al. Genetic alterations activating kinase and cytokine receptor signaling in high-risk acute lymphoblastic leukemia. Cancer Cell. 2012;22(2):153-166.

14. Hirabayashi S, Ohki K, Nakabayashi K, et al. ZNF384-related fusion genes define a subgroup of childhood $B$-cell precursor acute lymphoblastic leukemia with a characteristic immunotype. Haematologica. 2017;102(1):118-129.

15. Gu Z, Churchman M, Roberts K, et al. Genomic analyses identify recurrent MEF2D fusions in acute lymphoblastic leukaemia. Nat Commun. 2016;7:13331.

16. Mullighan CG, Goorha S, Radtke I, et al. Genome-wide analysis of genetic alterations in acute lymphoblastic leukaemia. Nature. 2007;446(7137):758-764.

17. Stanulla M, Dagdan E, Zaliova M, et al. IKZF1plus defines a new minimal residual disease-dependent very-poor prognostic profile in pediatric B-cell precursor acute lymphoblastic leukemia. J Clin Oncol. 2018;36(12):1240-1249.

18. Moorman AV, Enshaei A, Schwab C, et al. A novel integrated cytogenetic and genomic classification refines risk stratification in pediatric acute lymphoblastic leukemia. Blood. 2014;124(9):1434-1444.

19. Hamadeh L, Enshaei A, Schwab C, et al. Validation of the United Kingdom copy-number alteration classifier in 3239 children with B-cell precursor ALL. Blood Adv. 2019;3(2):148-157.

20. Sive Jl, Buck G, Fielding A, et al. Outcomes in older adults with acute lymphoblastic leukaemia (ALL): results from the international MRC UKALL XII/ECOG2993 trial. Br J Haematol. 2012;157(4):463-471.

21. Schwab CJ, Jones LR, Morrison H, et al. Evaluation of multiplex ligation-dependent probe amplification as a method for the detection of copy number abnormalities in B-cell precursor acute lymphoblastic leukemia. Genes Chromosomes Cancer. 2010;49(12):1104-1113.

22. Marks DI, Kirkwood AA, Rowntree CJ, et al. First analysis of the UKALL14 phase 3 randomised trial to determine if the addition of rituximab to standard induction chemotherapy improves EFS in adults with precursor B-ALL (CRUK/09/006). Blood.

2019;134(Suppl_1):739.

23. Moorman AV, Butler E, Barretta E, et al. Prognostic impact of chromosomal abnormalities and copy number alterations among adults with B-cell precursor acute lymphoblastic leukaemia treated on UKALL14. Blood. 2019;134(Suppl_1):288.

24. Mullighan CG, Miller CB, Radtke I, et al. BCR-ABL1 lymphoblastic leukaemia is characterized by the deletion of Ikaros. Nature. 2008;453(7191):110-114.

25. Tate JG, Bamford S, Jubb HC, et al. COSMIC: the catalogue of somatic mutations in cancer. Nucleic Acids Res. 2019;47(D1):D941-D947.

26. Holmfeldt L, Wei L, Diaz-Flores E, et al. The genomic landscape of hypodiploid acute lymphoblastic leukemia. Nat Genet. 2013;45(3):242-252.

27. Mühlbacher V, Zenger M, Schnittger S, et al. Acute lymphoblastic leukemia with low hypodiploid/near triploid karyotype is a specific clinical entity and exhibits a very high TP53 mutation frequency of 93\%. Genes Chromosomes Cancer. 2014;53(6):524-536.

28. Fröhling $S$, Scholl $C$, Levine RL, et al. Identification of driver and passenger mutations of FLT3 by high-throughput DNA sequence analysis and functional assessment of candidate alleles. Cancer Cell. 2007;12(6):501-513.

29. Jaiswal S, Fontanillas P, Flannick J, et al. Age-related clonal hematopoiesis associated with adverse outcomes. N Engl J Med. 2014;371(26):2488-2498.

30. Genovese G, Kähler AK, Handsaker RE, et al. Clonal hematopoiesis and blood-cancer risk inferred from blood DNA sequence. N Engl J Med. 2014;371(26):2477-2487.

31. Sim N-L, Kumar P, Hu J, et al. SIFT web server: predicting effects of amino acid substitutions on proteins. Nucleic Acids Res. 2012;40(W1):W452-W457.

32. Adzhubei IA, Schmidt S, Peshkin L, et al. A method and server for predicting damaging missense mutations. Nat Methods. 2010;7(4):248-249.

33. Benjamin K, Nicola G, Stefan S, et al. Loss-of-function but not dominant-negative intragenic IKZF1 deletions are associated with an adverse prognosis in adult BCR-ABL-negative acute lymphoblastic leukemia. Haematologica. 2017;102(10):1739-1747.

34. Marks DI, Paietta EM, Moorman AV, et al. T-cell acute lymphoblastic leukemia in adults: clinical features, immunophenotype, cytogenetics, and outcome from the large randomized prospective trial (UKALL XII/ECOG 2993). Blood. 2009;114(25):5136-5145.

35. Burmeister T, Schwartz S, Bartram CR, et al. Patients' age and $B C R-A B L$ frequency in adult B-precursor ALL: a retrospective analysis from the GMALL study group. Blood. 2008;112(3):918-919.

36. Safavi S, Paulsson K. Near-haploid and low-hypodiploid acute lymphoblastic leukemia: two distinct subtypes with consistently poor prognosis. Blood. 2017;129(4):420-423.

37. Tasian SK, Hurtz C, Wertheim GB, et al. High incidence of Philadelphia chromosome-like acute lymphoblastic leukemia in older adults with B-ALL. Leukemia. 2017;31(4):981-984.

38. Perez-Andreu V, Roberts KG, Harvey RC, et al. Inherited GATA3 variants are associated with $\mathrm{Ph}$-like childhood acute lymphoblastic leukemia and risk of relapse. Nat Genet. 2013;45(12):1494-1498.

39. Herold T, Baldus CD, Gökbuget N. Ph-like acute lymphoblastic leukemia in older adults. N Engl J Med. 2014;371(23):2235.

40. Fedullo AL, Messina M, Elia L, et al. Prognostic implications of additional genomic lesions in adult Philadelphia chromosome- 
positive acute lymphoblastic leukemia. Haematologica. 2019;104(2):312-318.

41. Moorman AV, Schwab C, Ensor HM, et al. IGH@ translocations, CRLF2 deregulation, and microdeletions in adolescents and adults with acute lymphoblastic leukemia. J Clin Oncol. 2012;30(25):3100-3108.

42. Clappier E, Auclerc MF, Rapion J, et al. An intragenic ERG deletion is a marker of an oncogenic subtype of B-cell precursor acute lymphoblastic leukemia with a favorable outcome despite frequent IKZF1 deletions. Leukemia. 2014;28(1):70-77.

43. Zhang J, McCastlain K, Yoshihara H, et al. Deregulation of DUX4 and ERG in acute lymphoblastic leukemia. Nat Genet. 2016;48(12):1481-1489.
44. Van der Meulen J, Sanghvi V, Mavrakis K, et al. The H3K27me3 demethylase UTX is a gender-specific tumor suppressor in Tcell acute lymphoblastic leukemia. Blood. 2015;125(1):13-21.

45. Ler LD, Ghosh S, Chai X, et al. Loss of tumor suppressor KDM6A amplifies PRC2-regulated transcriptional repression in bladder cancer and can be targeted through inhibition of EZH2. Sci Transl Med. 2017;9(378):eaai8312.

46. Hellemans J, Preobrazhenska O, Willaert A, et al. Loss-offunction mutations in LEMD3 result in osteopoikilosis, Buschke-Ollendorff syndrome and melorheostosis. Nat Genet. 2004;36(11):1213-1218.

47. Foà $R$, Bassan $R$, Vitale $A$, et al. Dasatinib-blinatumomab for $\mathrm{Ph}$-positive acute lymphoblastic leukemia in adults. N Engl $J$ Med. 2020;383(17):1613-1623. 